Nonlinear Processes in Geophysics, 12, 575-585, 2005

SRef-ID: $1607-7946 / \mathrm{npg} / 2005-12-575$

European Geosciences Union

(c) 2005 Author(s). This work is licensed

under a Creative Commons License.

\title{
Log-periodic behavior in a forest-fire model
}

\author{
B. D. Malamud ${ }^{1}$, G. Morein ${ }^{2}$, and D. L. Turcotte ${ }^{3}$ \\ ${ }^{1}$ Environmental Monitoring and Modelling Research Group, Department of Geography, King's College London, Strand, \\ London, WC2R 2LS, UK \\ ${ }^{2}$ Center for Computational Science and Engineering, University of California, Davis, CA 95616, USA \\ ${ }^{3}$ Department of Geology, University of California, Davis, CA 95616, USA
}

Received: 3 January 2005 - Revised: 29 March 2005 - Accepted: 30 April 2005 - Published: 9 June 2005

\begin{abstract}
This paper explores log-periodicity in a forest-fire cellular-automata model. At each time step of this model a tree is dropped on a randomly chosen site; if the site is unoccupied, the tree is planted. Then, for a given sparking frequency, matches are dropped on a randomly chosen site; if the site is occupied by a tree, the tree ignites and an "instantaneous" model fire consumes that tree and all adjacent trees. The resultant frequency-area distribution for the small and medium model fires is a power-law. However, if we consider very small sparking frequencies, the large model fires that span the square grid are dominant, and we find that the peaks in the frequency-area distribution of these large fires satisfy log-periodic scaling to a good approximation. This behavior can be examined using a simple mean-field model, where in time, the density of trees on the grid exponentially approaches unity. This exponential behavior coupled with a periodic or near-periodic sparking frequency also generates a sequence of peaks in the frequency-area distribution of large fires that satisfy log-periodic scaling. We conclude that the forest-fire model might provide a relatively simple explanation for the log-periodic behavior often seen in nature.
\end{abstract}

\section{Introduction}

This paper explores log-periodicity in a forest-fire cellularautomata model. Cellular-automata (CA) models are latticebased models which have simple "rules", but often exhibit complex behavior. At each time step, a series of nearestneighbour rules of interaction are applied, and individual cells are updated in the next step. A brief history of cellular automata is given by Sarkar (2000). A number of "simple" CA models have been shown to exhibit robust powerlaw statistics (e.g. for reviews see Turcotte, 1999; Malamud and Turcotte, 2000). Two examples of these include the sand-

Correspondence to: B. D. Malamud

(bruce@malamud.com) pile model and the forest-fire model, the latter which we will focus on in this paper.

A fractal distribution is a power-law distribution with a real exponent. If the exponent is taken to be a complex number, log-periodic behavior is obtained. In this paper we show that the peaks in the frequency-area distribution of large fires in the forest-fire model can satisfy log-periodic scaling to a good approximation. The possibility of complex scaling exponents and "log periodicity" in critical phenomena has been known for three decades (Nauenberg, 1975) with reviews of log-periodic variabilities given by Sornette $(1998,2002)$, Johansen and Sornette (2001), and Ide and Sornette (2002). We now briefly review a few specific studies that have proposed spatial and/or temporal log-periodic behavior, including (a) ruptures, (b) seismicity, (c) ground-water, (d) bronchial trees, (e) financial systems, and (f) models:

(a) Ruptures: Anifrani et al. (1995), Johansen and Sornette (2000), and Zhou and Sornette (2002) found that in rupture experiments there is a log-periodic relationship between the rate of acoustic emissions as a function of time, prior to the rupture of pressure vessels composed of carbon fibre reinforced resin.

(b) Seismicity: Saleur et al. (1996a) demonstrated a logperiodic increase in the cumulative Benioff strain as a function of time, where the strain is associated with regional earthquakes prior to the 17 October 1989 Loma Prieta (California) earthquake $(m=7.1)$. However, this work has been questioned by Huang et al. (2000b) and by Main et al. (2000). In other seismicity studies, Ouillon and Sornette (2000) presented data indicating a logperiodic increase in the rate of rockbursts as a function of time, in deep mines in South Africa. Finally, Huang et al. (2000a) argued in favour of log-periodic deviations from Omori's law in the temporal decay of aftershock activity.

(c) Ground water: Johansen et al. (2000) give evidence of log-periodic fluctuations as a function of time in 
ground-water chemistry prior to the 1995 Kobe earthquake. In other studies, Shibata et al. (2003) found logperiodic variations as a function of time in water levels prior to the 2000 eruption of the Usu Volcano, Japan.

(d) Bronchial trees: Schlesinger and West (1991) demonstrated a log-periodic dependence of mean branch diameter as a function of branch orders for bronchial trees.

(e) Financial time series: The temporal behavior of financial time series, including stock indices, exchange rates, and prices, have been argued by a number of authors to exhibit log-periodic fluctuations as a function of time (Feigenbaum and Freund, 1996; Sornette et al., 1996b; Sornette and Johansen, 1997; Johansen and Sornette 1999, 2001; Drozdz et al., 1999, 2003; Zhou and Sornette, 2003).

(f) Models: In addition to the "real" world, many theoretical models have also been shown to exhibit log-periodic behavior. For example, Blumenfeld and Ball (1991) modelled a propagating crack and found a spatial logperiodic distribution of corrugations on the crack as a function of the distance from the crack tip. In other rupture studies, Newman et al. (1995) and Sahimi and Arbabi (1996) have shown that models of rupture simulations exhibit log-periodic behaviour. In logistic map studies, Cavalcante et al. (2001) showed that bifurcation iterations exhibit log-periodic behavior in the rate of period-doubling on the route to chaos as a function of the value of the control parameter. Similarly, Canessa (2000) found log-periodic variations in a statistical birth-death model. Finally, Saleur et al. (1996b) has related log-periodicity to renormalization group analyses and Sornette et al. (1996a) with the structure of diffusion limited aggregation clusters.

As discussed above, a number of authors have proposed spatial and/or temporal log-periodic behavior in engineering applications, natural phenomena, social systems, and models. In this paper, we will examine log-periodic behaviour in the forest-fire model. We first review the sandpile and the forestfire CA models (Sect. 2), followed by examining the mathematics of log-periodicity and its application to the forestfire model (Sect. 3). We then show that the peaks in the frequency-area distribution of large forest-fire model fires satisfy log-periodic behavior (Sect. 4) and finally we advance a mean-field model as a general context within which to understand this log-periodic behavior (Sect. 5). We conclude that the forest-fire model might provide a relatively simple explanation for the log-periodic behavior often seen in nature.

\section{Two cellular automata models}

A classic example of a CA model that exhibits power-law frequency-size statistics of its "output", is the sandpile model introduced by Bak et al. (1988). In this model, a square array of boxes is considered and at each time step a particle is dropped into a randomly selected box. When a box accumulates four particles, they are redistributed to the four adjacent boxes, or in the case of edge boxes they are lost from the grid. Because the redistribution involves only the nearestneighbour boxes, it is a CA model. Redistributions of particles can lead to further instabilities with, at each time step, the possibility of avalanches of particles being lost from the grid. Each of the multiple redistributions during a time step contributes to the size of the model "avalanche". The size of an avalanche can be associated with the number of particles lost from the grid during the sequence of redistributions or by the number of boxes that participate in the redistributions. Extensive numerical studies of the "sandpile" model were carried out by Kadanoff et al. (1989). They found the noncumulative frequency-size distribution of avalanches to have a power-law distribution with a slope near unity. Bak et al. (1988) used the term "self-organized criticality" (SOC) to classify this type of scaling behavior (see Turcotte 1999 for a review of SOC). Lee and Sornette (2000) used a modified version of the sandpile model and obtained log-periodic scaling of avalanche magnitudes in time.

Another CA model that exhibits this type of scaling behavior is the forest-fire model, and is the subject of this paper. The forest-fire model that we use was introduced by Drossel and Schwabl (1992) and has been considered in great detail by Grassberger (2002). This forest-fire model consists of a square grid of sites. At each time step either a model tree or match is dropped on a randomly chosen site. A tree is "planted" only if the site is unoccupied. If a match is dropped on a cell with a tree, the tree ignites and a model fire consumes that tree and all adjacent (nondiagonal) trees. For a match dropped on an empty site nothing happens. The sparking frequency, $f$, is the inverse number of attempted tree drops on the square grid before a model match is dropped. If $1 / f=100$, there have been 99 attempts to plant trees (some successful, some unsuccessful) before a match is dropped at the 100th time step.

On average, the number of trees lost in "fires" is equal to the number of trees planted. However, the number of trees on the grid will fluctuate. The frequency-area distribution of model fires is a statistical measure of the behavior of the system. The noncumulative frequency-area distribution of small and medium model fires is fractal (power-law) with a slope of about 1.2 (Grassberger, 2002). The forest-fire model is probabilistic (stochastic) in that the sites are chosen randomly. It is a cellular-automata model in that only nearest-neighbour trees are ignited by a "burning" tree. In Sect. 4, we will present examples of some model fires and statistical results from our forest-fire model simulations.

Many variations on the basic forest-fire model described here have been proposed (Bak et al., 1992; Mossner et al., 1992; Henley, 1993; Christensen et al., 1993; Drossel and Schwabl, 1994; Johansen, 1994; Clar et al., 1996). One example of a variation is to use a stochastic distribution of intervals between match drops. Another example is the omission 

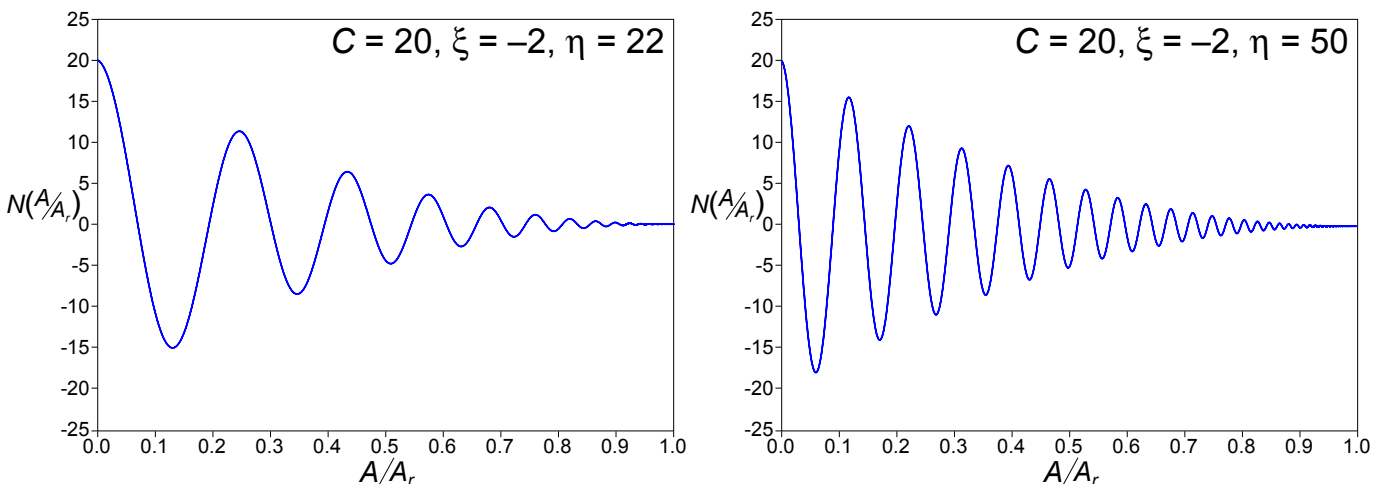

(a)

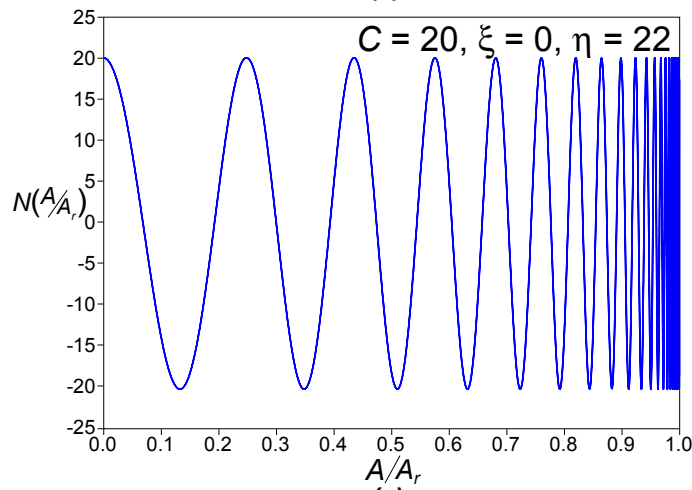

(c)

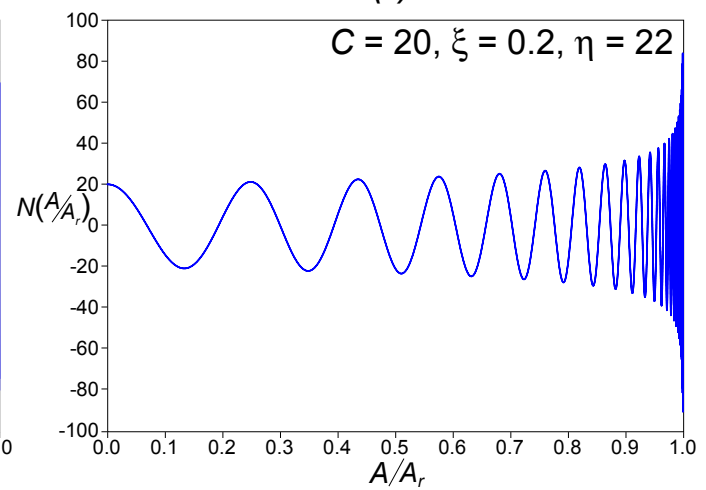

(d)

Fig. 1. The log-periodic equation $N\left(A / A_{r}\right)=C\left[1-\left(A / A_{r}\right)\right]^{-\xi} \cos \left\{-\eta \ln \left[1-\left(A / A_{r}\right)\right]\right\}$ from Eq. (2) is plotted on linear axes, $N\left(A / A_{r}\right)$ as a function of $A / A_{r}$. Shown in each of the four cases are the values chosen for the three constants $C$, $\eta$, and $\xi$. (i) The effect of changing $\eta$ : $C$ and $\xi$ remain the same, with $\eta=22$ in (a), $\eta=50$ in (b); the total number of crest-to-crest waves increases with increasing $\eta$. (ii) The effect of changing $\xi$ : $C$ and $\eta$ remain the same, with $\xi=-2$ in (a), $\xi=0$ in (c), and $\xi=0.2$ in (d). For $\xi<0$ in (a) the waveform amplitudes decrease as $A / A_{r}$ goes from 0 to 1 , compared to $\xi=0$ in (c) where the waveform amplitudes remain the same for $A / A_{r}$ from 0 to 1 , and finally $\xi>0$ in (d) where the waveform amplitudes increase as $A / A_{r}$ goes from 0 to 1 . For each of the four examples presented in this figure, the sequences of areas $A / A_{r}$ corresponding to the crests (local maxima, or peaks) of the waveforms are given by Eq. (3).

of time steps in which an attempt is made to plant a tree on an occupied site. However, in general, the frequency-area distributions of all models are similar. This is taken as evidence that the behavior is insensitive to the details of the models; the rules of the cellular-automata models can vary but the results are robust. We will now examine the mathematics of log-periodic scaling in some detail, before returning again to the forest-fire model in Sect. 4.

\section{Log-periodic scaling}

The concept of fractals was introduced by Mandelbrot (1967) in terms of statistical self-similarity. Given a set of objects or events with different areas, one standard measure (Turcotte, 1997) of fractality is the power-law dependence of number $N$ on area $A$,

$N(A) \sim A^{-D / 2}$

where $D$ is the fractal dimension. Log-periodic scaling follows directly from a complex fractal dimension, $D / 2=$ $\xi+i \eta$, with $i$ the square root of minus one, and $\xi$ and $\eta$ both real numbers. We again relate the number $N$ to the area $A$ with the result (Saleur et al., 1996a)

$$
\begin{aligned}
N(A) & =\operatorname{Re}\left\{C\left[1-\frac{A}{A_{r}}\right]^{-\xi-i \eta}\right\} \\
& =\operatorname{Re}\left\{C\left[1-\frac{A}{A_{r}}\right]^{-\xi} \exp \left(-i \eta \ln \left[1-\frac{A}{A_{r}}\right]\right)\right\} \\
& =C\left[1-\frac{A}{A_{r}}\right]^{-\xi} \cos \left(-\eta \ln \left[1-\frac{A}{A_{r}}\right]\right),
\end{aligned}
$$

where $R e$ stands for the real part, $C \geq 0$ is real and a constant, and $A_{r}$ is a reference value of $A$ with $A<A_{r}$ and both $A$ and $A_{r}$ positive and real.

To aid the reader in visualizing the log-periodic Eq. (2), in Fig. 1 we plot four examples on linear axes for $N\left(A / A_{r}\right)$ as a function of $A / A_{r}$. We keep $C=20$ constant in each of the four examples, and use three different values $\xi=-2.0$, $0.0,0.2$ and two values $\eta=22,50$. In each of the four graphs, Eq. (2) results in a quasi-periodic waveform, with the "period" (the horizontal distance between crests of the waveform) decreasing as $A / A_{r}$ goes from 0 to 1 . Figure 1 demonstrates the effect on the wave form if we change $\eta$ in the log-periodic relationship given in Eq. (2), with $\eta=22$ in 


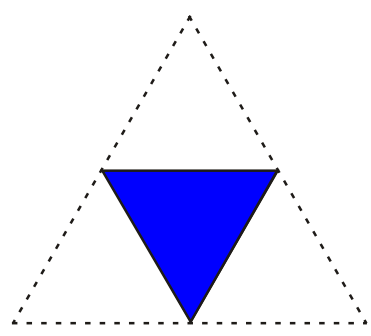

(a)

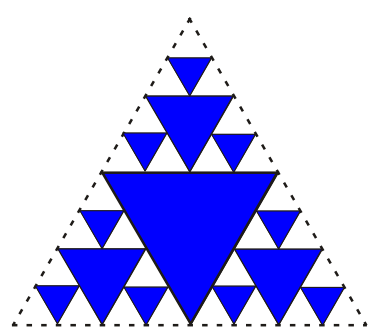

(c)

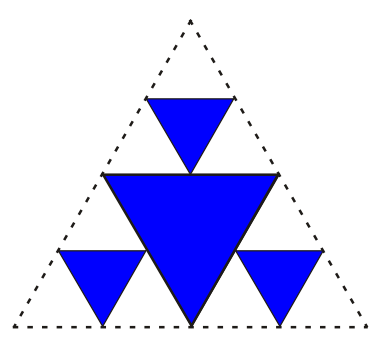

(b)

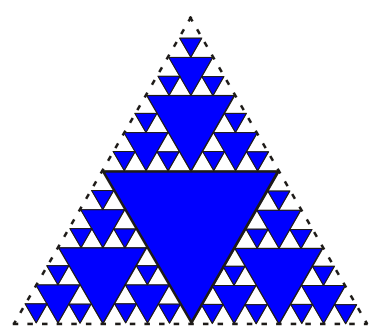

(d)
Fig. 2. Four orders of a fractal Sierpinsky gasket. The reference triangle formed by the dashed lines is equilateral and has reference area $A_{r}=1$. (a) At first order this "empty" reference triangle is divided into four equal-sized smaller triangles, each with area $A_{1}=1 / 4$. The middle triangle is retained (shaded), giving $N_{1}=1$ triangles at order one. This first order acts as a generator for each successive order, where in each case we go to the remaining "empty" triangles, divide each of them into four equal-sized smaller triangles, and retain (shade) the middle one. In the (b) second order we have added $N_{2}=3$ triangles each with area $A_{2}=1 / 16$, in (c) third order $N_{3}=9$ with $A_{3}=1 / 64$, and in (d) fourth order $N_{4}=27$ with $A_{4}=1 / 256$. The shaded areas satisfy the logperiodic sequence given in Eq. (4).

Fig. 1a, $\eta=50$ in Fig. $1 \mathrm{~b}$, and $C$ and $\xi$ remaining the same; we see the total number of crest-to-crest waves increases with increasing $\eta$. Figure 1 also demonstrates the effect on the waveform of changing the constant $\xi$ in Eq. (2), with $\xi=$ -2.0 (Fig. 1a), $\xi=0.0$ (Fig. 1c), $\xi=0.2$ (Fig. 1d), and $C$ and $\eta$ remaining the same. For $\xi<0$ (Fig. 1a) the waveform amplitudes decrease as $A / A_{r}$ goes from 0 to 1, compared to $\xi=0$ (Fig. 1c) where the waveform amplitudes remain the same for $A / A_{r}$ from 0 to 1 , and finally $\xi>0$ (Fig. 1d) where the waveform amplitudes increase as $A / A_{r}$ goes from 0 to 1 .

We note that Eq. (2) gives both positive and negative values of $N(A)$ as a function of $\eta$ and $A / A_{r}$. Thus modifications to this equation must be made where only positive quantities for $N(A)$ are considered. This can be done if $N(A)$ is a combination of the real fractal given in Eq. (1) and the complex fractal given in Eq. (2). However, in the application considered here, we are only interested in the sequence of maxima for $N(A)$ as given by Eq. (2), e.g. the sequence of values of $A / A_{r}$ in Figs. 1a to $1 \mathrm{~d}$ that correspond to the crests (the local maxima) for each waveform. We obtain this sequence by letting $x=\left[1-\left(A / A_{r}\right)\right]$ in Eq. (2) and setting $d N(A) / d x=0$, giving (Turcotte, 1997, Eq. 15.42)

$\frac{A_{k}}{A_{r}}=1-\exp \left[-\frac{1}{\eta} \tan ^{-1}\left(\frac{\xi}{\eta}\right)-\frac{2 k \pi}{\eta}\right]$,

$k=0,1,2,3, \ldots$

with $A_{k}$ the sequence of areas $A$ corresponding to the maxima for $N(A)$. The spacing between successive values of $A_{k}$ approaches zero as $k$ becomes large and $A_{k}$ approaches $A_{r}$. For the log-periodic behaviour considered in the rest of this paper, we will use Eq. (3).

We next consider a simple geometrical construction that gives the sequence of log-periodic values given by Eq. (3). Consider the fractal Sierpinsky gasket illustrated in Fig. 2. We take the triangular area within the dashed lines to be a reference area with $A_{r}=1$. The fractal relation (Eq. 1) can be applied to the number of triangles $N_{k}$ with area $A_{k}$. In (a) $(k=1)$ we have $N_{1}=1, A_{1}=1 / 4$, in (b) $(k=2)$ we have added $N_{2}=3, A_{2}=1 / 16$, in (c) $(k=3) N_{3}=9$, $A_{3}=1 / 64$, and in (d) $(k=4) N_{4}=27, A_{4}=1 / 256$. From Eq. (1) we find that the fractal dimension $D=\ln 3 / \ln 2 \approx$ 1.585 .

We now consider the shaded areas in each of the constructions illustrated in Fig. 2. In (a) we have $A_{1} / A_{r}=1-$ $(3 / 4)=0.250$, in (b) we have $A_{2} / A_{r}=1-(3 / 4)^{2} \approx 0.438$, in (c) we have $A_{3} / A_{r}=1-(3 / 4)^{3} \approx 0.578$, and in (d) we have $A_{4} / A_{r}=1-(3 / 4)^{4} \approx 0.684$. This result can be generalized to arbitrary order $k$ with the result

$\frac{A_{k}}{A_{r}}=1-\left(\frac{3}{4}\right)^{k}, \quad k=0,1,2,3, \ldots$

The log-periodic set of areas given in Eq. (3) reduces to the set of areas given in Eq. (4) if we take $\xi=0$ and $\eta=2 \pi / \ln (4 / 3) \approx 22$. This can also be seen in Fig. 1c where Eq. (2) is plotted with $\xi=0$ and $\eta=22$; the sequence of values for $A / A_{r}$ corresponding to local maxima of $N$ is $A / A_{r}=0,0.250,0.438,0.578,0.684,0.763,0.822, \ldots$, or in other words the sequence of values given in Eq. (4). Since our original construction is a scale-invariant fractal construction, it is not surprising that the sequence of total areas at each step of the construction obeys a scale-invariant log-periodic law.

The log-periodic sequence of areas corresponding to peaks, as given in Eq. (3), can also be used to predict the reference area and subsequent areas in the sequence. If any three successive values of $A_{k}$ are observed, i.e. $A_{i+1}, A_{i+2}$, $A_{i+3}$, then the reference value, $A_{r}$, is given by

$A_{r}=\frac{A_{i+2}^{2}-A_{i+1} A_{i+3}}{2 A_{i+2}-A_{i+1}-A_{i+3}}$.

If we take $A_{j+1}=A_{i+1}+A^{\prime}, A_{j+2}=A_{i+2}+A^{\prime}, A_{j+3}=$ $A_{i+3}+A^{\prime}$, then Eq. (5) is also satisfied for $A_{j+1}, A_{j+2}$ and $A_{j+3}$. Thus Eq. (5) is independent of origin and can be used for any sequence of three $A_{k}$. In addition, the fourth value in a sequence, $A_{i+4}$, is related to the previous three values, $A_{i+1}, A_{i+2}, A_{i+3}$, by

$A_{i+4}=\frac{A_{i+2}^{2}+A_{i+3}^{2}-A_{i+1} A_{i+3}-A_{i+2} A_{i+3}}{A_{i+2}-A_{i+1}}$. 
We will utilize Eqs. (5) and (6) to show that peaks in the frequency-area distribution of our model fires satisfy logperiodic statistics to a good approximation.

\section{Forest-fire model results}

The forest-fire model that we consider was described in the introduction. Our forest-fire model is dependent on the size of the square grid, $N_{g}$, and the sparking frequency, $f$. In our model, fires stop at the edge of the grid and do not wrap around to the other side. A simulation is run for $N_{S}$ time steps and the number of fires $N_{F}$ with area $A_{F}$ is determined. The area is the number of trees that burn in a fire. Examples of four typical fires during a run are given in Fig. 3. In these examples the grid size is $128 \times 128\left(N_{g}=16384\right), 1 / f=$ 2000, and fires with $A_{F}=5,51,505$ and 5327 trees are illustrated.

Noncumulative frequency-area statistics for the model fires are given in Fig. 4. The number of fires per time step with area $A_{F}, N_{F} / N_{S}$, is given as a function of $A_{F}$. In Fig. 4a results are given for a grid size $N_{g}=128 \times 128=16384$ cells and three sparking frequencies, $1 / f=125,500$ and 2000. The small and medium model fires correlate well with the power-law relation

$\frac{N_{F}}{N_{S}} \sim A_{F}^{-\alpha}$

with $\alpha=1.0$ to 1.2 . This is equivalent to the fractal relation (Eq. 1).

For large fires, the frequency-area distribution in Fig. 4 deviates significantly from a straight-line, such that there is an upper termination to the power-law distribution. In Fig. 4a the deviation is downwards for $1 / f=125$ and begins at $A_{F} \approx 800$, or $1 / 20$ the size of the grid. At smaller firing frequencies, the large model fires become more numerous, resulting in an upward deviation from the small fire powerlaw behavior. For the smallest firing frequency shown in Fig. $4 a, 1 / f=2000$, this deviation begins at approximately $A_{F}=2000$, or $1 / 8$ the grid size. When the sparking frequency is very small, large fires become dominant. This can be explained on physical grounds. With a large sparking frequency (for example $1 / f=125$ ) trees burn before large clusters can form. If the sparking frequency is very small (for example $1 / f=2000$ ) clusters form that span the entire grid before ignition occurs.

An example of a model fire that spans the grid is illustrated in Fig. $4 \mathrm{~d}\left(A_{F}=5327\right)$. For the simulation with firing frequency $1 / f=2000$ and grid size $128 \times 128$, we studied the spanning characteristics of different size model fires and found no fires with size $A_{F}<2000$ span the grid, 50\% of the fires at $A_{F}=3800$ span the grid, and all fires with $A_{F}>5300$ span the grid. The threshold area at which clusters begin to span a grid will be explored further in Sect. 5, in the context of "percolating" clusters.

In order to examine in more detail the frequency-area distributions of these large clusters we have considered even

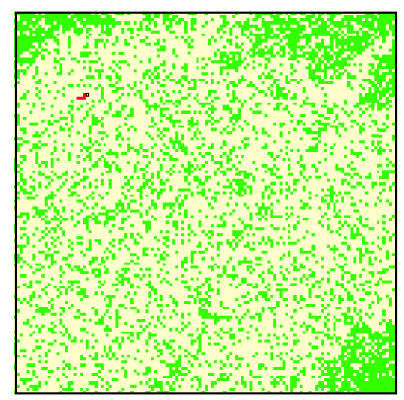

(a) $A_{F}=5$ trees

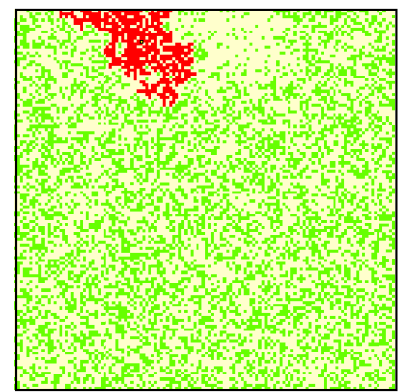

(c) $A_{F}=505$ trees

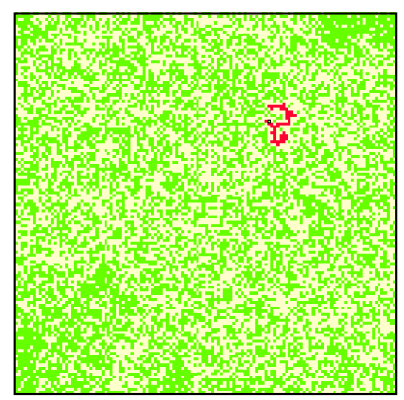

(b) $A_{F}=51$ trees

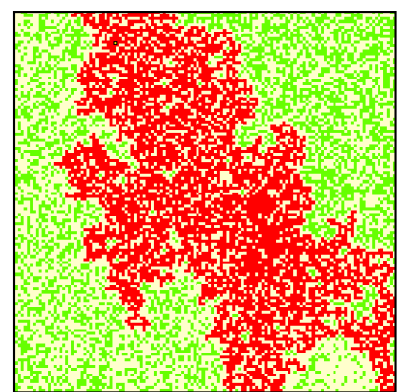

(d) $A_{F}=5,327$ trees
Fig. 3. Four examples of typical fires in the forest-fire model are given (figure after Malamud et al., 1998). This run was carried out on a $128 \times 128$ grid with $1 / f=2000$. The red regions are the model fires. The green regions are unburned trees. The white regions are unoccupied grid points. The areas $A_{F}$ of the four model fires are given. The largest model fire is seen to span the entire grid.

smaller firing frequencies. Frequency-area statistics for large fires are given in Figs. $4 \mathrm{~b}$ to $4 \mathrm{~d}$ for a $128 \times 128$ grid size and sparking frequencies $1 / f=5000,10000$, and 20000 . It is seen that the model fires cluster at well defined fire areas. For example, for $1 / f=20000$ the clusters peak at $A_{F 1^{\prime}}=11400, A_{F 2^{\prime}}=14975, A_{F 3^{\prime}}=15970$, and $A_{F 4^{\prime}}=16260$. We use $1^{\prime}, 2^{\prime}, 3^{\prime}, 4^{\prime}$ as subscripts for the successive peaks; these are "any" successive four peaks. The total number of grid sites available is $N_{g}=128^{2}=16384$. The model fires that we observe for the three firing frequencies shown in Figs. $4 \mathrm{~b}$ to $4 \mathrm{~d}$ are tabulated in Table 1a.

We now utilize Eqs. (5) and (6) derived in the context of log-periodic scaling, in order to see whether the observed forest-fire model peaks satisfy log-periodic behavior. We hypothesize that the $A_{r}$ given in Eq. (5) is the total number of grid sites available, $A_{r}=N_{g}=16384$. We substitute areas corresponding to sequences of three observed peaks, $A_{F 1^{\prime}}$, $A_{F 2^{\prime}}, A_{F 3^{\prime}}$, into Eq. (5) and determine $A_{r}$ for each of the observed sequences. For our observed peak sequences we also use $A_{F 2^{\prime}}, A_{F 3^{\prime}}, A_{F 4^{\prime}}$, and $A_{F 3^{\prime}}, A_{F 4^{\prime}}, A_{F 5^{\prime}}$. The results calculated for $A_{r}$ are given in Table $1 \mathrm{~b}$. It is seen that all values of $A_{r}$ are close to the hypothesized value $A_{r}=N_{g}=16384$. Next, we again use areas corresponding to sequences of three observed peaks, $A_{F 1^{\prime}}, A_{F 2^{\prime}}, A_{F 3^{\prime}}$, and $A_{F 2^{\prime}}, A_{F 3^{\prime}}, A_{F 4^{\prime}}$, substitute these two sets of observed values into Eq. (6), and thus derive the next value predicted for each sequence. The predicted values of $A_{F 4^{\prime}}$ and $A_{F 5^{\prime}}$ (Table 1c) are in good agreement with the observed values (Table 1a). 
Table 1. (a) Observed peaks in forest-fire model areas*. (b) Predictions of grid size based on sequences of three observed peaks ${ }^{\dagger}$. (c) Predictions of $A_{F 4^{\prime}}, A_{F 5^{\prime}}$ based on sequences of three observed peaks $\ddagger$.

\begin{tabular}{ccccccc}
\hline (a) & $1 / f$ & $A_{F 1^{\prime}}$ & $A_{F 2^{\prime}}$ & $A_{F 3^{\prime}}$ & $A_{F 4^{\prime}}$ & $A_{F 5^{\prime}}$ \\
\hline & 5000 & 11390 & 12810 & 13760 & 14450 & 14920 \\
& 10000 & 11400 & 13800 & 14975 & 15600 & 15975 \\
& 20000 & 11400 & 14975 & 15970 & 16260 & - \\
\hline (b) & $1 / f$ & $\left(A_{F 1^{\prime}}, A_{F 2^{\prime}}, A_{F 3^{\prime}}\right)$ & $\left(A_{F 2}, A_{F 3^{\prime}}, A_{F 4^{\prime}}\right)$ & $\left(A_{F 3^{\prime}}, A_{F 4^{\prime}}, A_{F 5^{\prime}}\right)$ & & \\
\cline { 1 - 4 } & 5000 & 15756 & 16410 & 15710 & & \\
& 10000 & 16102 & 16310 & 16358 & & \\
& 20000 & 16353 & 16379 & - & & \\
\cline { 1 - 4 } (c) & $1 / f$ & $A_{F 4^{\prime}}$ & $A_{F 5^{\prime}}$ & & & \\
\cline { 1 - 4 } & 5000 & 14395 & 14966 & & & \\
& 10000 & 15550 & 15932 & & & \\
& 20000 & 16247 & - & & & \\
\hline
\end{tabular}

* (a) The peaks in the fire areas $A_{F 1^{\prime}}, A_{F 2^{\prime}}, A_{F 3^{\prime}}, A_{F 4^{\prime}}, A_{F 5^{\prime}}$, illustrated in Figs. $4 \mathrm{~b}$ to $4 \mathrm{~d}$ for $1 / f=5000$, 10000 , and 20000 are tabulated. Note that peaks at higher values of $A_{F}$ are present but have not been tabulated.

${ }^{\dagger}$ (b) The values of $A_{r}$ calculated from Eq. (5) are given for sequences of three fire areas from (a) We associate $A_{r}$ with the grid area $N_{g}=128 \times 128=16384$ and find good agreement.

$\$$ (c) The predicted values of $A_{F 4^{\prime}}$ and $A_{F 5^{\prime}}$ using Eq. (6) are given for observed sequences of three fire areas from (a). The predicted values are in good agreement with the observed $A_{F 4^{\prime}}$ and $A_{F 5^{\prime}}$ given in (a).

\section{Mean-field model}

In the last section, we showed that for one grid size $N_{g}=$ $128 \times 128$ and three small firing frequencies, $1 / f=5000$, 10000,20000 , that the frequency-area distribution of the model fires strongly peak at specific fire areas. These peaks have been illustrated in Figs. 4b to 4d, and tabulated in Table 1a. We now extend our studies to smaller and larger grid sizes, $N_{g}=64 \times 64$ and $N_{g}=256 \times 256$, and firing frequencies from $1 / f=1000$ to 160000 . The values of the observed peaks for these studies are tabulated in Table 2. Our objective is to provide a model to explain the distribution of peaks.

The peaks we wish to study occur only at very small values of the firing frequency. For small firing frequencies the loss of trees occurs primarily in large grid-spanning fires. After a very large fire we assume it is a good approximation to set the number of trees on the grid, $A_{t}$, to be zero and take this to be the time origin, thus $A_{t}=0$ at $t=0$. In this limit the behavior of the forest-fire model is identical to the behavior of the site-percolation model (Stauffer and Aharony, 1992). In this case it is appropriate to make a mean-field approximation for the increase in the number (total area) $A_{t}$ of trees on the grid with time. Although $t$ increases in discrete time steps of $\Delta t=1$, we can approximate this increase by the continuum relation

$$
\frac{d A_{t}}{d t}=1-\frac{A_{t}}{N_{g}} .
$$

Initially $A_{t}$, the number of trees on the grid, is small and $d A_{t} / d t \approx 1$; therefore, a tree is planted at every time step. Subsequently the probability that a tree is planted at a time step is $\left[1-\left(A_{t} / N_{g}\right)\right]$. Solving Eq. (8) with $A_{t}=0$ and $t=0$ we obtain

$\frac{A_{t}}{N_{g}}=1-\exp \left(-\frac{t}{N_{g}}\right)$.

In other words, without fires the density of trees on the grid $A_{t} / N_{g}$ initially increases rapidly with time $t$, with the rate of increase decreasing exponentially and an asymptotic limit of 1 tree per cell (a fully occupied grid).

Matches are dropped at times $t=k / f, k=1,2,3,4, \ldots$. Using this expression and $A_{F k}=A_{t}$ in Eq. (9), then the set of fire sizes corresponding to these match drops is

$$
\frac{A_{F k}}{N_{g}}=1-\exp \left(-\frac{k}{f N_{g}}\right), \quad k=1,2,3,4, \ldots
$$

Note that the succession of theoretical peaks $A_{F k}=A_{F 1}$, $A_{F 2}, A_{F 3}, A_{F 4}, \ldots$ are not necessarily the same set of successive observed peaks as discussed in the last section $A_{F 1^{\prime}}$, $A_{F 2^{\prime}}, A_{F 3^{\prime}}, A_{F 4^{\prime}}, \ldots$; this is discussed further below. The fire areas $A_{F k}$ approach the grid size $N_{g}$ as $k$ becomes large. Associating the grid size $N_{g}$ in Eq. (10) with the reference area $A_{r}$ in Eq. (3) and taking $\xi=0$ and $\eta=2 \pi f N_{g}$, the forest-fire model area peaks $A_{F k}$ in Eq. (10) correspond to 

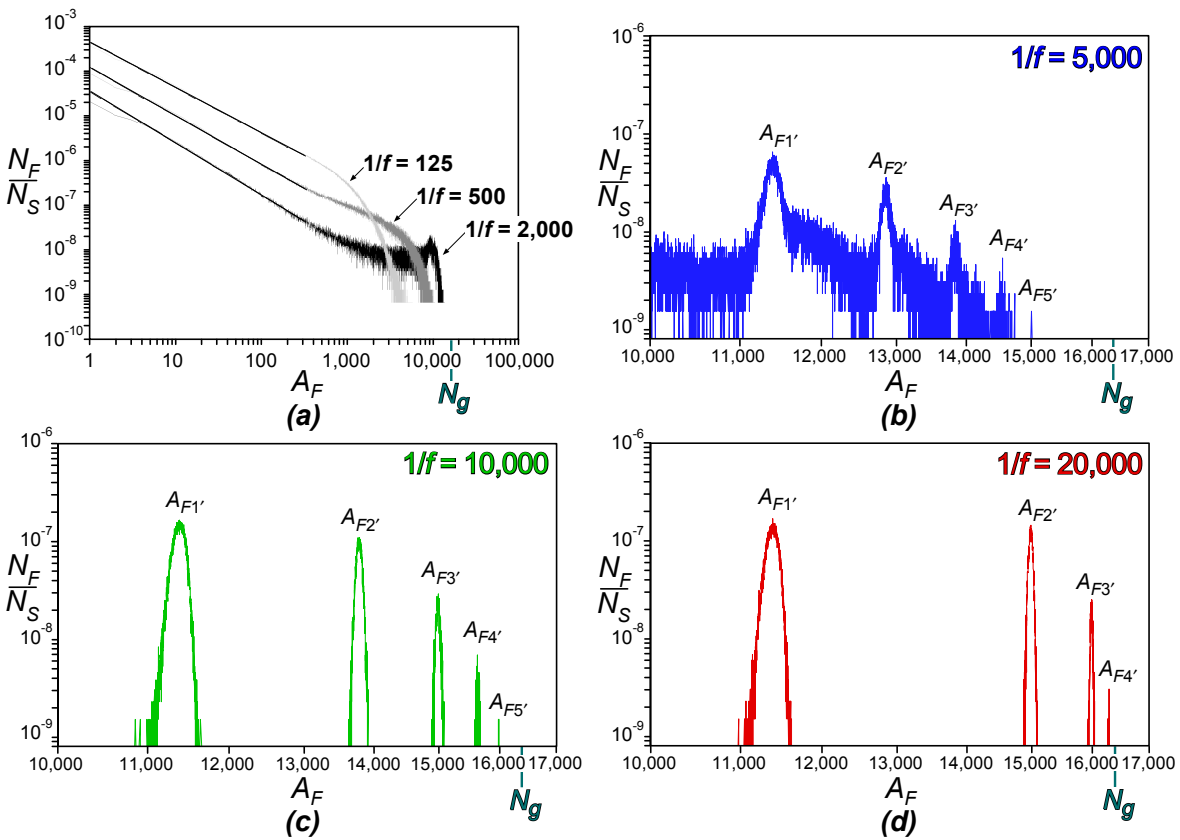

Fig. 4. Frequency-area statistics of the forest-fire model. (a) The number of fires per time step $N_{F} / N_{S}$ with area $A_{F}$ (number of lattice cells in the "fire"), is given as a function of $A_{F}$ (Fig. 4a after Malamud et al., 1998). Results are given for a grid with $N_{g}=128 \times 128$, and firing frequencies $1 / f=125,500$, and 2000. To further investigate the behavior of the distribution for the very large fires, we again use grid size $N_{g}=128 \times 128$ and focus in on the area from $A_{F}=10000$ to $A_{F}=17000$ for three different firing frequencies $(\mathbf{b}) 1 / f=5000,(\mathbf{c})$ $1 / f=10000$, and (d) $1 / f=20000$. Marked in each figure are the observed peaks that occur, $A_{F 1^{\prime}}, A_{F 2^{\prime}}, A_{F 3^{\prime}}, \ldots$, and the location on the horizontal axis for $A_{F}=N_{g}$.

the areas $A_{k}$ given in Eq. (3). This simple model predicts a log-periodic distribution of fire peaks.

In Table 2 we give a wide range of observed peaks $\left(A_{F}\right.$ observed) obtained from forest-fire model simulations. Grid sizes $64 \times 64\left(N_{g}=4096\right), 128 \times 128\left(N_{g}=16384\right)$, and $256 \times 256\left(N_{g}=65536\right)$ have been considered. Firing frequencies range from $1 / f=1000$ to 160000 . Numbers of observed peaks range from two to seven. The observed values previously given in Table 1 a for $1 / f=5000$ and 20000 are also included. Using the values of $N_{g}$ and $f$ used in each simulation, theoretical predicted values of peak areas $A_{F k}$ are obtained from the log-periodic mean-field relation (Eq. 10) for various values of $k$. These are given in Table 2 as $A_{F k}$ theoretical, with $k$ given in brackets. It is seen that there is excellent agreement between the mean field predictions and the values observed in the forest-fire model simulations.

The results given in Table 2 are also illustrated in Fig. 5 . The mean-field results given in Eq. (10) can be rewritten as

$$
-\ln \left(1-\frac{A_{F k}}{N_{g}}\right)=\frac{k}{f N_{g}}, \quad k=1,2,3,4, \ldots
$$

In Fig. 5, the dependence of $1 / f$ on $-\ln \left[1-\left(A_{F k} / N_{g}\right)\right]$ is shown for $N_{g}=128 \times 128$ and $k=1$ to 12 using solid straight lines. Also included in Fig. 5 as data points are the observed peaks from our forest-fire model simulations as given in Table 2 for $N_{g}=128 \times 128$ and five values of $1 / f$. It is seen that the sequence of areas corresponding to peaks in the simulations closely agree with the sequence of areas given by increasing integer values of $k$ in our mean-field prediction from Eq. (11).

We now address $1^{\prime}, 2^{\prime}, 3^{\prime}, \ldots$ in sequences of observed areas $A_{F 1^{\prime}}, A_{F 2^{\prime}}, A_{F 3^{\prime}}, \ldots$, not necessarily corresponding to $k=1,2,3, \ldots$ in theoretical areas $A_{F k}$. Specifically, consider $N_{g}=128 \times 128=16384$ cells, and $1 / f=5000$, as given in Table 1a. Comparing with Table 2, we find $A_{F 1^{\prime}}$ (i.e. the first observable peak area) corresponds to $A_{F 4}$ (i.e. the fourth theoretical peak area), $A_{F 2^{\prime}}$ corresponds to $A_{F 5}$, etc. The peaks associated with $k=1,2,3$ are "missing". To explain this we introduce the concept of percolating clusters.

A percolating cluster corresponds to a cluster of trees that span the grid. At any given time, more than one spanning cluster on the grid can only occur on very rare occasions. From studies of site percolation we can conclude that the "threshold" for a spanning cluster occurring is $p_{c}=$ $A_{t c} / N_{g}=0.59275$ (Stauffer and Aharony, 1992) where $A_{t c}$ is the number of trees in the spanning clusters. Substituting $A_{t c}$ for $A_{F k}$ in Eq. (11), and $A_{t c} / N_{g}=0.59275$, we find that at the percolation threshold, $-\ln \left[1-\left(A_{t c} / N_{g}\right)\right]=0.8983$. This value is included in Fig. 5 as a dashed vertical line and in Table 2, for each grid size, the threshold spanning cluster area is given. In the region to the left of the dashed vertical line in Fig. 5, no peaks in area are observed in forest-fire model simulations. Peaks only occur for clusters that span the grid and when the density of trees on the grid is greater than the critical density for grid spanning clusters. We note that in 
Table 2. Theoretical peaks for $A_{F k}$ compared to observed peaks*.

\begin{tabular}{|c|c|c|c|c|c|c|c|c|c|}
\hline$N_{g}$ & $1 / f$ & $\begin{array}{l}\begin{array}{l}\text { Spanning } \\
\text { Cluster }\end{array} \\
\end{array}$ & $\begin{array}{l}A_{F k} \text { Theore } \\
\left(A_{F} \text { Obser }\right.\end{array}$ & $\begin{array}{l}\text { tical }[\boldsymbol{k}] \\
\text { ved) }\end{array}$ & & & & & \\
\hline 4096 & 1000 & 2428 & 2888 [5] & 3149 [6] & 3354 [7] & $3515[8]$ & 3641 [9] & $3739[\mathbf{1 0}]$ & 3817 [11] \\
\hline \multirow[t]{9}{*}{$(64 \times 64)$} & & & 2845 & 3146 & 3350 & 3513 & 3660 & 3744 & 3835 \\
\hline & 1250 & 2428 & $2888[4]$ & 3205 [5] & 3440 [6] & 3612 [7] & $3739[8]$ & 3833 [9] & $3902[\mathbf{1 0}]$ \\
\hline & & & 2840 & 3200 & 3440 & 3615 & 3743 & 3816 & 3890 \\
\hline & 2000 & 2428 & 3149 [3] & $3515[4]$ & 3739 [5] & 3877 [6] & 3962 [7] & & \\
\hline & & & 3140 & 3521 & 3743 & 3883 & 3965 & & \\
\hline & 5000 & 2428 & 2888 [1] & 3739 [2] & 3991 [3] & $4065[4]$ & 4087 [5] & & \\
\hline & & & 2850 & 3747 & 3993 & 4065 & 4088 & & \\
\hline & 10000 & 2428 & 3739 [1] & $4065[2]$ & 4093 [3] & & & & \\
\hline & & & 3742 & 4066 & 4093 & & & & \\
\hline 16384 & 3000 & 9712 & 10923 [6] & 11837 [7] & $12597[8]$ & 13231 [9] & $13759[\mathbf{1 0}]$ & $14198[11]$ & $14564[12]$ \\
\hline \multirow[t]{11}{*}{$(128 \times 128)$} & & & 10600 & 11750 & 12597 & 13227 & 13745 & 14218 & 14588 \\
\hline & 5000 & 9712 & 11550 [4] & 12822 [5] & 13759 [6] & 14449 [7] & 14958 [8] & 15333 [9] & $15609[\mathbf{1 0}]$ \\
\hline & & & 11390 & 12810 & 13760 & 14450 & 14920 & 15317 & 15628 \\
\hline & 8000 & 9712 & 12597 [3] & $14060[4]$ & 14958 [5] & 15509 [6] & & & \\
\hline & & & 12560 & 14080 & 14960 & 15530 & & & \\
\hline & 14000 & 9712 & 13418 [2] & 15122 [3] & 15847 [4] & 16155 [5] & & & \\
\hline & & & 13400 & 15130 & 15860 & 16150 & & & \\
\hline & 20000 & 9712 & 11550 [1] & 14958 [2] & 15963 [3] & $16260[4]$ & & & \\
\hline & & & 11400 & 14975 & 15970 & 16260 & & & \\
\hline & 100000 & 9712 & 16347 [1] & 16384 [2] & & & & & \\
\hline & & & 16348 & 16384 & & & & & \\
\hline \multirow{8}{*}{$\begin{array}{c}65536 \\
(256 \times 256)\end{array}$} & 20000 & 38846 & 46201 [4] & 51287 [5] & $55034[6]$ & 57796 [7] & $59832[8]$ & & \\
\hline & & & 45560 & 51250 & 55100 & 57770 & 59900 & & \\
\hline & 40000 & 38846 & 46201 [2] & 55034 [3] & 59832 [4] & 62438 [5] & $63853[6]$ & & \\
\hline & & & 45630 & 55100 & 59880 & 62430 & 63850 & & \\
\hline & 80000 & 38846 & 46201 [1] & 59832 [2] & 63853 [3] & 65040 [4] & $65390[5]$ & & \\
\hline & & & 45630 & 59875 & 63855 & 65065 & 65400 & & \\
\hline & 160000 & 38846 & 59832 [1] & 65040 [2] & 65493 [3] & & & & \\
\hline & & & 59800 & 65040 & 65494 & & & & \\
\hline
\end{tabular}

*Included in this table are the values predicted $\left(A_{F k}\right.$ theoretical) using the mean-field results given in Eq. (10) with the value of $\boldsymbol{k}$ used given in square brackets. Also included are the observed peaks ( $A_{F}$ observed) obtained from forest-fire simulations for three grid sizes, $64 \times 64\left(N_{g}=4096\right)$ $128 \times 128\left(N_{g}=16384\right)$ and $256 \times 256\left(N_{g}=65536\right)$, and a variety of firing frequencies $1 / f=1000$ to 160000 .

$\dagger$ The spanning cluster is equal to $0.59275 N_{g}$ and is the critical value below (or nearby) which peaks in $A_{F}$ are not observed.

actuality, theoretical peaks slightly greater than this percolating threshold cluster, although they occur, can be difficult to observe.

As an example, we take the case discussed previously $\left(N_{g}=128 \times 128\right.$ and $\left.1 / f=5000\right)$. In Fig. 5 , we see from the row of diamonds, that the peaks corresponding to $k=1$, 2,3 are "missing". As another example, consider the data for $1 / f=3000$ and $N_{g}=128 \times 128$ in Table 2 . The smallest observed peak for fire areas is at $A_{F 1^{\prime}}=10600$. This corresponds to $-\ln \left[1-\left(A_{F} / N_{g}\right)\right]=1.041$ which is above the percolation threshold of 0.8983 , and agrees with the theoretical predicted peak $A_{F 6}=10923$ from Eq. (11) using $k=6$,
$1 / f=3000$ and $N_{g}=128 \times 128$. The predicted peaks for $k=1, \ldots, 5$ do not occur, as the location of these peaks are less than the spanning clusters, so they do not show up as peaks. Peaks in the distribution of fires occur only when the density of trees on the grid is greater than the critical density for grid spanning clusters.

We have shown that the areas of large fires in a forest-fire model correlate with a log-periodic scaling when the sparking frequency is very small. We have given a mean-field analysis that reproduces the model results with considerable accuracy. The exponential approach of tree area to the total grid area coupled with periodic sparking leads to this behav- 
ior. The large model fires peak only when the density of trees on the grid exceeds the percolation threshold for tree clusters that span the grid. These spanning clusters are responsible for the peaks that we see in our distribution of fire areas, with at any given time only one spanning cluster on the grid. If the first match dropped after the percolation threshold is reached hits a tree, a fire corresponding to the peak with the smallest area is observed. If the first match misses a tree then the spanning cluster grows exponentially as shown in Eq. (9). If the second match hits a tree, a fire in the peak with the next largest peak area is observed, and so forth. The exponential growth of cluster areas asymptotically approaching the size of the grid, combined with a periodic (or near periodic) sparking frequency, are necessary conditions for robust logperiodic behavior.

\section{Conclusions}

Many natural phenomena are associated with "avalanches" that satisfy power-law (fractal) frequency-magnitude distributions to a good approximation. Examples include landslides (Guzzetti et al., 2002; Malamud et al., 2004), earthquakes (Aki, 1981; Turcotte and Malamud, 2002), and wildfires (Malamud et al., 1998, 2005; Ricotta et al., 1999). These examples are also closely associated with simple cellular automata that are said to exhibit self-organized criticality (Bak et al., 1988). Landslides with the sandpile model, earthquakes with the slider-block mode, and wildfires with the forest-fire model.

In this paper we have studied the behavior of the forestfire model when the firing frequency is very small. Trees are planted until they form a large cluster that spans the square grid of planting sites. These large clusters are "burned" when matches are dropped at large prescribed intervals. We have focused our attention on the frequency-area distribution of these very large fires. This distribution is characterized by a series of very well defined peaks which become more closely spaced with increasing fire size. We have shown that these peaks satisfy log-periodic scaling to a very good approximation. A log-periodic distribution can be obtained by taking a power-law (fractal) distribution and making the power-law exponent (the fractal dimension) a complex number.

We have also shown that a very simple mean-field model produces the log-periodic peaks seen in our forest-fire simulations. Without any fires the forest-fire model is identical to the site percolation model, where the number of trees (area) on the grid initially increases rapidly with time ( 1 tree per time step), with the rate of increase decreasing exponentially as the grid fills up. If trees are then destroyed (burned) at equally spaced time intervals, the areas have a log-periodic distribution of fire sizes.

As discussed in the introduction, log-periodic distributions are often seen in nature and in numerical simulations. The studies presented here illustrate circumstances in which logperiodicity can be expected. First a characteristic size is required. For the forest-fire model, this is the area of the grid.

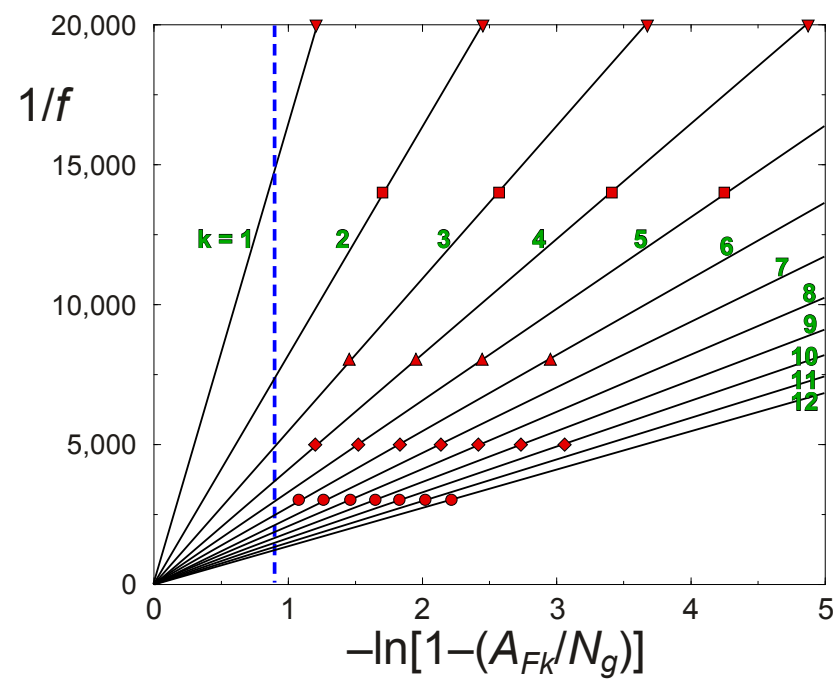

Fig. 5. The predicted mean-field dependence of the firing frequency $1 / f$ is given as a function of $-\ln \left[1-\left(A_{F k} / N_{g}\right)\right]$. Solid straight lines are from $-\ln \left[1-\left(A_{F k} / N_{g}\right)\right]=\left(\left(k / f N_{g}\right)\right)$ (Eq. 11) for twelve different values of $k$ and $N_{g}=128 \times 128$. The data points (triangles, squares, diamonds and circles) are the observed peaks ( $A_{F}$ observed, in Table 2 ) for $1 / f=3000,5000,8000,14000$, 20000 and $N_{g}=128 \times 128$. The agreement between "observed" and "predicted" values, also seen in Table 2 , is clearly illustrated in this figure. The vertical dashed line at $-\ln \left[1-\left(A_{F k} / N_{g}\right)\right]=$ 0.89833 represents the percolation model threshold at which the spanning cluster area is $A_{t c}=0.59275 \times N_{g}=A_{F k}$. To the left of this vertical line, peaks in the frequency-area distribution are not observed for the forest-fire model.

Second, the trigger for the "avalanches" must be periodic or near periodic. In the forest-fire model, the periodic triggers are the regular spaced match drops. We conclude that the forest-fire model might provide a relatively simple explanation for the log-periodic behavior often seen in nature.

Acknowledgements. The contributions of author DLT were partially supported by NSF Grant NO ATM 0327558 and the contributions of author BDM were partially supported by the UK NERC/EPSRC Grant NER/T/S/2003/00128. We also thank the two referees, S. Hergarten and one person who remained anonymous, for their very helpful comments.

Edited by: G. Zöller

Reviewed by: S. Hergarten and another referee

\section{References}

Aki, K.: A probabilistic synthesis of precursory phenomena, in: Earthquake Prediction, edited by: Simpson, D. W. and Richards, P. G., Maurice Ewing Series 4, American Geophysical Union: Washington, D. C., 566-574, 1981.

Anifrani, J. C., Le Floch, C., Sornette, D., and Souillard, B.: Universal log-periodic correction to renormalization group scaling for rupture stress prediction from acoustic emissions, J. Phys. I France, 5, 631-638, 1995. 
Bak, P., Tang, C., and Wiesenfeld, K.: Self-organized criticality, Phys. Rev. A, 38, 364-374, 1988.

Bak, P., Chen, K., and Tang, C.: A forest-fire model and some thoughts on turbulence, Phys. Let. A, 147, 297-300, 1992.

Blumenfeld, R. and Ball, R. C.: Onset of scale-invariant pattern in growth processes: the cracking problem, Physica A, 177, 407415, 1991.

Canessa, E.: Stochastic theory of log-periodic patterns, J. Phys. A, 33, 9131-9140, 2000.

Cavalcante, H. L. D. D., Vasconcelos, G. L., and Leite, J. R. R.: Power law periodicity in the tangent bifurcations of the logistic map, Physica A, 295, 291-296, 2001.

Christensen, K., Flyvbjerb, H., and Olami Z.: Self-organized critical forest-fire model: Mean-field theory and simulation results in 1 to 6 dimensions, Phys. Rev. Let., 71, 2737-2740, 1993.

Clar, S., Drossel, B., and Schwabl, F.: Forest fires and other examples of self-organized criticality, J. Phys. Condensed Matter, 8, 6803-6824, 1996.

Drossel, B. and Schwabl, F.: Self-organized critical forest-fire model, Phys. Rev. Let., 69, 1629-1632, 1992.

Drossel, B. and Schwabl, F.: Formation of space-time structure in a forest-fire model, Physica A, 204, 212-229, 1994

Drozdz, S., Ruf, F., Speth, J., and Wojcik, W.: Imprints of logperiodic self-similarity in the stock market, Eur. Phys. J. B, 10, 589-593, 1999.

Drozdz, S., Grummer, F., Ruf, F., and Speth, J.: Log-periodic selfsimilarity: an emerging financial law?, Physica A, 324, 174-182, 2003.

Feigenbaum, J. A. and Freund, P. G. O.: Discrete scale invariance in stock markets before crashes, Int. J. Mod. Phys. B, 10, 3737 3745, 1996.

Grassberger, P.: Critical Behavior of the Drossel-Schwabl Forest Fire Model, New J. Phys., 4, 17.1-17.15, 2002.

Guzzetti, F., Malamud, B. D., Turcotte, D. L., and Reichenbach, P.: Power-law correlations of landslide areas in central Italy, Earth Plan. Sci. Lett., 195, 169-183, 2002.

Henley, C. L.: Statics of a "self-organized" percolation model, Phys. Rev. Let., 71, 2741-2744, 1993.

Huang, Y., Johansen, A., Lee, M. W., Saleur, H., and Sornette, D.: Artifactual log-periodicity in finite size data: relevance for earthquake aftershocks, J. Geophys. Res., 105, 25 451-25 471, 2000 a.

Huang, Y., Saleur, H., and Sornette, D.: Reexamination of log periodicity observed in the seismic precursors of the 1989 Loma Prieta earthquake, J. Geophys. Res., 105, 28 111-28 123, $2000 \mathrm{~b}$.

Ide, K. and Sornette, D.: Oscillatory finite-time singularities in finance, population and rupture, Physica A, 307, 63-106, 2002.

Johansen, A.: Spatio-temporal self-organization in a model of disease spreading, Physica D, 78, 186-193, 1994.

Johansen, A. and Sornette, D., Modeling the stock market prior to large crashes, Eur. Phys. J. B, 9, 167-174, 1999.

Johansen, A. and Sornette, D.: Critical ruptures, Eur. Phys. J. B, 18, 163-181, 2000.

Johansen, A. and Sornette, D.: Finite-time singularity in the dynamics of the world population, economic and financial indices, Physica A, 294, 465-502, 2001.

Johansen, A., Saleur, H., and Sornette, D.: New evidence of earthquake precursory phenomena in the 17 January 1995 Kobe earthquake, Japan, Eur. Phys. J. B, 15, 551-555, 2000.

Kadanoff, L. P., Nagel, S. R., Wu, L., and Zhou, S. M.: Scaling and universality in avalanches, Phys. Rev. A, 39, 6524-6533, 1989.

Lee, M. W. and Sornette, D.: Novel mechanism for discrete scale invariance in sandpile models, Eur. Phys. J. B, 15, 193-197, 2000.
Malamud, B. D., and Turcotte D. L.: Cellular-automata models applied to natural hazards, IEEE Comp. Sci. Eng., 2, 42-51, 2000.

Malamud, B. D., Morein, G., and Turcotte, D. L.: Forest fires: an example of self-organized critical behavior, Science, 281, 1840 1842, 1998.

Malamud, B. D., Turcotte, D. L., Guzzetti, F., and Reichenbach, P.: Landslide inventories and their statistical properties, Earth Surf. Proc. Landforms, 29, doi:10.1002/esp.1064, 687-711, 2004.

Malamud, B. D., Millington, J. D. A., and Perry G. L. W.: Characterizing wildfire regimes in the USA, Proc. Nat. Acad. Sci., 102, 4694-4699, 2005.

Main, I. G., O’Brien, G., and Henderson, J. R.: Statistical physics of earthquakes: comparison of distribution exponents for source area and potential energy and the dynamic emergence of logperiodic energy quanta, J. Geophys. Res., 105, 6105-6126, 2000.

Mandelbrot, B.: How long is the coast of Britain? Statistical self-similarity and fractional dimension, Science, 156, 636-638, 1967.

Mossner, W. K., Drossel, B., and Schwabl, F.: Computer simulations of the forest-fire model, Physica A, 190, 205-217, 1992.

Nauenberg, M.: Scaling representation for critical phenomena, J. Phys. A, 8, 925-928, 1975.

Newman, W. I., Turcotte, D. L., and Gabrielov, A. M.: Log-periodic behavior of a hierarchical failure model with applications to precursory seismic activation, Phys. Rev. E., 52, 4827-4835, 1995.

Ouillon, G. and Sornette, D.: The concept of "critical earthquakes" applied mine rockbursts with time-to-failure analysis, Geophys. J. Int., 143, 454-488, 2000.

Ricotta, C., Avena, G., and Marchetti M.: The flaming sandpile: self-organized criticality and wildfires, Ecol. Model., 119, 7377, 1999.

Sahimi, M. and Arbabi, S.: Scaling laws for fracture of heterogeneous materials and rock, Phys. Rev. Let., 77, 3689-3692, 1996.

Saleur, H., Sammis, C. G., and Sornette, D.: Discrete scale invariance, complex fractal dimensions, and log-periodic fluctuations in seismicity, J. Geophys. Res., 101, 17 661-17 677, 1996a.

Saleur, H., Sammis, C. G., and Sornette, D.: Renormalization group theory of earthquakes, Nonlin. Proc. Geophys., 3, 102109, 1996b, SRef-ID: 1607-7946/npg/1996-3-102.

Sarkar, P.: A brief history of cellular automata, ACM Computing Surveys, 32, 80-107, 2000.

Schlesinger, M. F. and West, B. J.: Complex fractal dimension of the bronchial tree, Phys. Rev. Let., 67, 2106-2108, 1991.

Shibata, T., Matsumoto, N., and Akita, F.: Fluctuation in groundwater level prior to the critical failure point of the crustal rocks, Geophys. Res. Let., 30, 1, art. no. 1024 , doi:10.1029/2002GL016050, 2003.

Sornette, D.: Discrete-scale invariance and complex dimension, Phys. Reports, 297, 239-270, 1998.

Sornette, D.: Predictability of catastrophic events: material rupture, earthquakes, turbulence, financial crashes, and human birth, Proc. Nat. Acad. Sci., 99, 2522-2529, 2002.

Sornette, D. and Johansen, A.: Large financial crashes, Physica A, 245, 411-422, 1997.

Sornette, D., Johansen, A., Arneodo, A., Muzy, J. F., and Saleur, H.: Complex fractal dimensions describe the hierarchical structure of diffusion-limited-aggregate clusters, Phys. Rev. Let., 76, 251254, 1996a.

Sornette, D., Johansen, A., and Bouchaud, J. P.: Stock market crashes, precursors and replicas, J. Phys. I France, 6, 167-175, 1996 b.

Stauffer, D. and Aharony, A.: Introduction to Percolation Theory, 
2nd ed., Taylor and Francis, London, 1992.

Turcotte, D. L. Fractals and Chaos in Geology and Geophysics, 2nd ed., Cambridge University Press, Cambridge, 1997.

Turcotte, D. L.: Self-organized criticality, Reports on Progress in Physics, 62, 1377-1429, 1999.

Turcotte, D. L. and Malamud, B. D.: Earthquakes as a complex system, in: International Handbook of Earthquake and Engineering Seismology, edited by: Lee, W. H. K, Kanamori, H., Jennings, P. C., and Kisslinger, C., Academic Press: London, 209-227, 2002.
Zhou, W. X. and Sornette, D.: Generalized q analysis of logperiodicity: applications to critical ruptures, Phys. Rev. E, 66, art. no. 046111, 8 p., 2002.

Zhou, W. X. and Sornette, D.: Evidence of a worldwide stock market log-periodic anti-bubble since mid-2000, Physica A, 330, 543-583, 2003. 